\title{
Intermédialités
}

Histoire et théorie des arts, des lettres et des techniques

Intermediality

History and Theory of the Arts, Literature and Technologies

\section{Le spectateur hors jeu : investigation ludique du réseau interférentiel}

\section{Sébastien Babeux}

Numéro 9, printemps 2007

Jouer

Playing

URI : https://id.erudit.org/iderudit/1005531ar

DOI : https://doi.org/10.7202/1005531ar

Aller au sommaire du numéro

Éditeur(s)

Centre de recherche sur l'intermédialité

ISSN

1705-8546 (imprimé)

1920-3136 (numérique)

Découvrir la revue

Citer cet article

Babeux, S. (2007). Le spectateur hors jeu : investigation ludique du réseau interférentiel. Intermédialités / Intermediality, (9), 79-98.

https://doi.org/10.7202/1005531ar
Résumé de l'article

Afin de considérer le fait de " jouer » un texte ou un film, le lecteur-spectateur doit en reconstituer l'aire de jeu. Cette reconstitution est nécessairement imparfaite, généralement étroite, mais elle peut également, au contraire, sous l'action de certains croisements intertextuels, se voir élargie. Nous étudions ce type de croisements, suivant les réflexions de Michel Serres, comme interférences. Asignifiante, l'interférence n'appelle pas à une interprétation juste : tout effort interprétatif se trouve à dépasser ses cadres, à surinterpréter. Elle place le spectateur dans une position qui lui permet de remanier le système signifiant qui lui est proposé. Autant au sens ludique qu'au sens mécanique, il y a jeu dans la brèche qu'opère l'interférence et qu'investit le spectateur, par pur plaisir encyclopédique ou par investigation herméneutique honnête. 


\title{
Le spectateur hors jeu: investigation ludique du réseau interférentiel
}

\author{
Sébastien BabeuX
}

- xaminant et analysant le jeu chez l'enfant, la réflexion de Donald Winni- cott s'ordonne autour d'une question centrale - où joue-t-on? - posant une spatialité propre et primordiale à l'activité ludique, un espace potentiel, ne se situant ni tout à fait dans le réel, ni tout à fait dans la psyché du joueur ${ }^{1}$. Le jeu posséderait donc un lieu. Ainsi, pour considérer le fait de jouer (dans) un système narratif (littéraire ou cinématographique), il est nécessaire d'aborder celui-ci, au moins à un niveau métaphorique, comme un espace. Or, l'approche intertextuelle marque d'emblée une spatialisation débordant l'espace textuel, resituant le texte à l'intérieur d'un espace plus vaste, en tant que «sous-ensemble d'un ensemble plus grand qui est l'espace des textes appliqués dans notre ensemble²». Réseau plus ou moins étendu, cet ensemble plus grand, l'intertexte, comprend un nombre varié de textes liés entre eux de différentes façons. Parmi ces liaisons, nous identifions deux grands groupes: 1) les relations textuelles concrètes mettent en relation effective un nombre fixe et déterminé de textes (c'est le cas par exemple de la citation, de l'allusion et de la parodie); 2) les relations textuelles non concrètes mettent quant à elles en relation un nombre variable et indéterminé de textes, dans une espèce de flottement intertextuel (c'est le cas par exemple du pastiche, du lieu commun et du cliché). Le premier met en place un réseau intertextuel centré, au sein duquel le texte tient place de noyau's . Le second, plus près d'une

1. Voir Donald Winnicott, Playing and Reality, London, Tavistock Publications, 1971.

2. Julia Kristeva, Sèméiotikè: recherches pour une sémanalyse, Paris, Éditions du Seuil, coll. «Tel quel», 1969, p. 194 .

3. Voir Mikhail Iampolski, The Memory of Tiresias. Intertextuality and Film, Los Angeles, University of California Press, 1998, p. 36 : «Intertextuality does not imply a confused and mysterious accumulation of influences, but the work of transformation and assimilation of several texts performed by a centering text which retains its position of leadership in meaning. » (nous soulignons) 
conception barthésienne de l'intertextualité où les croisements sont proprement irrepérables ${ }^{4}$, propose un réseau dépourvu de centre, où le texte tient plutôt lieu de pivot. L'interférence, que nous postulons à partir des écrits de Michel Serres et qui correspond à une relation (asignifiante) du second groupe, nous permettra ici de repenser de façon originale l'espace textuel selon une conception ludique du texte, marquant une frontière dans l'aire de jeu, une zone qui serait hors jeu sans pour autant être hors-du-jeu. Nous verrons dans un premier temps de quelle façon le texte lui-même se présente comme un espace propice au jeu, puis en quoi les relations textuelles concrètes qu'il entretient agissent comme un élargissement de cet espace. Finalement, nous examinerons comment un croisement interférentiel provoque un éclatement de cet espace et sa reformulation par le lecteur-spectateur.

Le concept d'interférence nous permettra donc de combler un manque dans la théorisation de l'intertextualité et d'aborder les croisements que le spectateur projette sur l'œuvre, mais qui ne lui appartiennent pas en propre. Si la réflexion de Michel Serres fut employée de différentes façons au sein des études sur le texte et le discours 5 , son application à l'approche intertextuelle du texte (et plus précisément, du film) est tout à fait originale et permet de mieux comprendre la dynamique d'échange et de circulation à l'œuvre entre les textes, ainsi que de déhiérarchiser le réseau intertextuel. Elle permet de faire un pont entre les deux solitudes caractérisant la majorité des travaux portant sur l'intertextualité: d'un côté, les théoriciens étudiant l'intertextualité comme typologie de relations textuelles concrètes, se penchant sur des exemples précis tout en laissant de côté de façon commode les relations non concrètes; de l'autre, ceux s'intéressant à l'intertexte comme multiplication indéterminable et inidentifiable de croisements tout en réduisant à l'embarrassante étude des sources les travaux se penchant, eux, sur des relations concrètes. Jonathan Culler notait déjà l'absurdité de cette dichotomie dans The Pursuit of Signs, précisant que Kristeva elle-même passait librement, dans Sèméiotikè, d'un côté à l'autre de ce gouffre creusé par ses disciples ${ }^{6}$. Le concept d'interférence réconcilie ces deux approches, en permettant non plus de les considérer tour à tour, mais en les liant, en proposant une nouvelle forme de croisement empruntant à l'une comme à l'autre. Notons d'entrée de jeu que s'il est relativement aisé de schématiser les relations textuelles concrètes, il est au contraire impossible de rendre compte de la constante mouvance du réseau

4. Voir Roland Barthes, «De l'œuvre au texte», dans Le bruissement de la langue. Essais critiques, vol. 4, Paris, Éditions du Seuil, 1984, p. 73.

5. Voir par exemple le numéro de Protée consacré aux interférences (Protée, vol. 24, $\mathrm{n}^{\circ}$ 2, automne 1996).

6. Voir Jonathan Culler, The Pursuit of Signs, Ithaca, Cornell University Press, 1981 , p. 106. 
interférentiel - le schéma que nous proposons ici, à partir d'une reformulation du système de communication de Claude Shannon, ne pourra donc être considéré que comme une illustration ponctuelle et incomplète de ce réseau.

\section{UN SYSTÈME SIGNIFIANT}

Axiome pour certains (les lecteurs de Christian Metz par exemple), il nous faut néanmoins, afin que nos propositions puissent produire du sens, poser ce postulat premier : le film, le «texte», s'exposant à la libre interprétation du lecteur-spectateur, est un système signifiant clos, rigide et fixé sur un support matériel7. Ce postulat n'implique d'aucune façon - et il est important de le comprendre - que nous prêchions ici en faveur d'une bonne lecture, d'une interprétation univoque du système, assimilable à une quelconque intentionnalité de son architecte (l'instance autoriale $\left.{ }^{8}\right)$. Ainsi, la phrase «C'était comme si personne n’avait entendu. » peut être interprétée d'innombrables façons : chacun de ses éléments a une résonance particulière, un champ paradigmatique propre dans l'encyclopédie de l'interprète; chacune de ses combinaisons générant des associations que l'on ne saurait retracer, dans le réseau intertextuel convoqué comme dans les expériences antérieures du lecteur. Cette phrase renvoie néanmoins à un système signifiant fixe qui, malgré l'«ouverture» interprétative de tout énoncé, demeure, comme système, clos et limité - au sens où il ne comprend pas le mot «bruit ${ }^{9}$ », au sens où n'y figure aucune citation de l'œuvre de Blaise Pascal. La réception du système signifiant requiert une compétence minimale (ici, une connaissance sommaire de la langue française), et c'est seulement une fois perçu, en tout ou en partie, que ce système (fixe et limité) «s'ouvre » aux interprétations multiples. Bien sûr, on ne peut recevoir un énoncé sans l'interpréter, et si cette interprétation, aussi

7. Les expérimentations du film interactif n'échappent pas à cette constatation: même lorsque l'ensemble de ses possibilités narratives n'est pas actualisé au cours d'un visionnement, le système demeure fixe, limité, bien qu'une part de sa matérialité (la totalité de ses images et de ses sons) demeure alors latente.

8. Nous utiliserons architecte et instance autoriale pour traiter, non pas de l'«auteur», mais de la «stratégie textuelle». Voir Umberto Eco, Lector in fabula. Le rôle du lecteur ou la coopération interprétative dans les textes narratifs, trad. Myriem Bouzaher, Paris, Éditions Grasset \& Fasquelle, 1979, p. 76: «l'auteur n’est autre qu'une stratégie textuelle capable d'établir des corrélations sémantiques. »

9. Le lecteur peut très bien considérer le «bruit» comme étant implicite et nécessaire à la compréhension de la phrase — ce serait là (entre autres) retrancher la possibilité «comprendre» du paradigme du lexème «entendre »; ce serait là, donc, une interprétation du système signifiant, qui lui se présente sans «bruit». 
excentrique soit-elle, ne peut être fondamentalement impossible ou inacceptable ${ }^{1 \circ}$, on peut se demander - et c'est ce que nous ferons ici - si la réception elle-même n'aurait pas été parasitée par un bruit (ajouté); si donc c'est le système signifiant tel qu'il se présentait qui fut l'objet de l'interprétation ou si on n'aurait pas un peu, consciemment ou non, joué avec les données.

\section{UN TERRAIN DE JEU}

Considérant le schéma du système de communication de Claude Shannon ${ }^{11}$, nous y trouvons déjà - l'exemple n'était pas innocent - ce bruit qui, se lovant dans le canal de l'échange, fait en sorte que le signal émis et le signal reçu ne sont sans doute jamais tout à fait les mêmes:

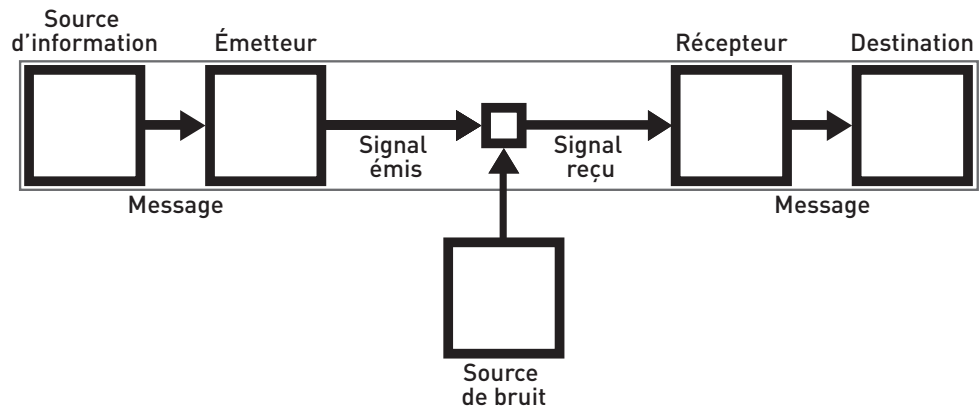

Fig. 1: Le système de communication de Claude Shannon (schéma)

Notre intention ici n'est aucunement d'emprunter ou d'ajouter quoi que ce soit à la théorie de l'information, mais simplement de souligner que l'on retrouve dans la proposition de Shannon une évidente spatialisation de la transmission et de la réception des messages. Le canal de transmission que met en place le schéma de Shannon ne nous intéresse guère au sens purement technique, par contre sa transposition sur les canaux de la réception artistique et de l'interprétation est pour nous plus féconde. Cette transposition est certes limitée (ne serait-ce que parce qu'elle implique un système binaire et un récepteur passif) et

10. " $[N]$ o reading, however outlandish it might appear, is inherently an impossible one. (Stanley Fish, Is There a Text in This Class? The Authority of Interpretive Communities, Cambridge, Londres, Harvard University Press, 1980, p. 347)

11. Claude E. Shannon et Warren Weaver, The Mathematical Theory of Communication, Urbana, University of Illinois Press, 1949. 
fait sans doute partie des abus qu'aura subis cette approche ${ }^{12}$, par contre la métaphore spatiale du passage du sens entre le système signifiant et l'interprète est encore aujourd'hui largement répandue dans les études littéraires et cinématographiques. On y qualifiera par exemple le processus cognitif de l'interprétation de parcours, réseau, ou autre «sentier interprétatif'13», le texte lui-même y étant bien souvent approché comme un lieu. «[L]e texte comme terrain de jeu ${ }^{14}$ », bien sûr, mais plus simplement le texte comme lieu devant être traversé (un champ, un itinéraire, un labyrinthe) - traversée étant habituellement linéaire et concrète, correspondant à «l'avancée matérielle dans le volume lu, dans l'espace ludique $^{15}$ ». Tourner une page, visionner une scène, ce serait alors faire un pas de plus dans cet espace à explorer. Cette directivité, mise en place autant par la matérialité du livre ${ }^{16}$ que par la stratégie textuelle établie, est celle du walkthrough (solution) du jeu vidéo: elle aménage un itinéraire précis permettant de traverser le texte sans exploration superflue, sans retour en arrière inutile (laissant dans l'ombre la part inutile du monde diégétique). Si, dans le jeu, elle permet au joueur de ne pas s'égarer inutilement et de restreindre son expérience ludique à la bonne solution, dans le texte, cette directivité est généralement considérée comme génératrice de l'expérience ludique elle-même (via la distribution calculée et filtrée de l'information) ${ }^{17}$. Dans les deux cas, elle constitue un appauvrissement de l'expérience ludique totale ou potentielle et la reconstitution de son tracé ne rend pas compte de l'espace à explorer dans sa globalité; elle est

12. Voir Pierre-Jean Lancry, «Les limites de la théorie de l'information », in Théorie de l'information et économie, Paris, Éditions ECONOMICA, 1982, p. 53-57.

13. Bernard Perron, «Un indice pour ouvrir le jeu», Cinémas, «Cinélekta 3», vol. 10, $\mathrm{n}^{\circ} 1$, automne 1999, p. 108.

14. Umberto Eco, Les limites de l'interprétation, trad. Myriem Bouzaher, Paris, Éditions Grasset et Fasquelle, 1992 [1990], p. 46.

15. Michel Picard, «La lecture comme jeu», Poétique, n 58, mars 1984, p. 257.

16. Umberto Eco donne dans L'ouvre ouverte l'exemple du Livre de Mallarmé comme système signifiant «ouvert» (ouverture n'étant toujours que partielle) et dont la matérialité ne poserait pas une directivité aussi rigide. Voir Umberto Eco, L'œuvre ouverte, trad. Chantal Roux de Bézieux, Éditions du Seuil, Paris, 1965 [1962], p. 26-28.

17. Voir André Gardies, «Le pouvoir ludique de la focalisation », Protée, «Le point de vue fait signe», vol. 16, $\mathrm{n}^{\text {os }} 1-2$, hiver-printemps 1988, p. 143: «La régulation du savoir est précisément ce qui permet au jeu fictionnel de se maintenir: spectateur, je ne puis construire mes hypothèses de "lecture" sans prendre appui sur ce que je sais à chaque moment du récit en cours. » Il ne faut pas se leurrer, cette distribution régulée de l'information est également présente dans le jeu vidéo et là aussi génératrice, en partie (et de façon plus perceptible au sein des jeux s'appuyant sur une construction narrative plus élaborée), de la possibilité de jouer. 
strictement limitée à l'espace donné (ou imposé) par l'instance autoriale. Il nous apparaît donc nécessaire de diviser le «lieu » de l’interprétation en deux zones, comme un terrain de jeu : 1) le système signifiant en lui-même, le tracé du livre, du film (le volume dans son ensemble) - ce système correspond au signal émis; 2) le système signifiant résultant de la réception du système premier, objet de l'interprétation, construction de l'interprète avec ses retranchements et ses ajouts au système premier - ce système correspond quant à lui au signal reçu. Il y aurait ainsi une zone qui serait globalement en-jeu (tout ce qui appartient au volume) et le parcours réel de la lecture-spectature, dont les débordements constitueraient la zone hors jeu. Entre les deux, bruits et interférences faisant dévier le sens du «message» et risquant à tout moment de provoquer ces ajouts et débordements.

\section{4}

\section{UNE ZONE EN-JEU}

Sans accorder d'importance à la quête du sens littéral d'un texte, il est nécessaire d'admettre qu'à la source de toute interprétation, de toute lecture, se trouve un objet premier doté d'une existence objective : un objet lu. Cet objet, autonome au sens où il se présente comme entier, est constitué, matériellement, d'un nombre fini d'éléments. On peut en décomposer la somme selon différentes échelles : la phrase «C'était comme si personne n'avait entendu. » comprend un nombre fini de mots, de verbes, de phonèmes, de consonnes, de lettres, etc. Le film a une matérialité plus évanescente, il est difficile d'en répertorier ou d'en chiffrer les sons, mais il en contient tout de même une certaine quantité, comme il contient une quantité fixe de plans, de photogrammes, d'acteurs, de raccords sur le regard, de pièces de musique, etc. Il y a donc là une construction stable, qui se donne à voir et à déchiffrer. Or, cet objet ne recouvre pas la totalité de l'espace délimité par l'instance autoriale: du système signifiant, nous n'avons, dans sa matérialité, que le noyau, que le centre. Si ce centre est un lieu, il est proprement la distance franchie par l'avancée matérielle dans le volume lu (ou le film visionné). Il est également l'espace privilégié de la majorité des analogies entre les différents appareils narratifs (principalement la littérature et le cinéma) et le jeu, que nous résumerons ici - de façon restreinte - par ces quelques lignes d’André Gardies :

[...] postuler le caractère fondamentalement ludique du fait filmique et de la participation spectatorielle, c'est, dans le même temps, supposer un fonctionnement narratif placé sous le signe du déploiement stratégique. Le visionnement et la lecture d'un film s'assimilent au déroulement d'une partie mettant en présence deux joueurs: l'énonciateur et l'énonciataire. De cela, et à propos du texte littéraire, Umberto Eco donne une première idée lorsqu'il montre, dans Lector in fabula, comment le narrateur procède en avançant par propositions à l'adresse du lecteur qui, lui, répond 
par un ensemble d'hypothèses. Une sorte de compétition («l'agôn») s’instaure entre eux, fondée sur une mobilisation de l'imaginaire ${ }^{18}$.

Cette conception de la lecture-spectature comme procédé ludique d'investigation (propositions/hypothèses; confirmation/infirmation; ou le modèle plus répandu questions/réponses), s’appuie elle aussi sur une spatialisation du texte et sur son exploration, généralement linéaire ${ }^{19}$ et sans débordement. Cet isolement dans l'œuvre est certes favorable à l'analogie ludique, le jeu nécessitant son propre espace, coupé de la réalité (l'espace potentiel du jeu de l'enfant; les mondes possibles des jeux du lecteur). Ainsi, pour la majorité des théoriciens, l'absorption (diégétique) est primordiale à la participation ludique du lecteur-spectateur de l'œuvre: celui-ci doit perdre de vue la conception du système pour se perdre dans sa substance, comme l'enfant doit perdre de vue le monde réel pour se perdre dans son jeu. «[C] elui qui refuse brutalement d'acquiescer à l'illusion proposée, qui rappelle au garçon qu'il n'est pas un vrai détective ${ }^{20}$ » est considéré briseur de jeu. Celui qui refuse de jouer le jeu, c'est-à-dire d'être dans le (monde du) jeu, qui demeure à l'extérieur, est hors-du-jeu : c'est le cas par exemple du spécialiste observant le jeu de l'enfant; c'est le cas également du théoricien observant le mécanisme ludique d'un texte ${ }^{21}$.

Suivant cette approche, le jeu narratif se présente comme un ensemble de pistes, d'indices et de devinettes, mis en place par l'instance narrative à l'intention du joueur (lecteur-spectateur) qui, lui, aura beau jeu de se laisser berner par les embûches narratives (et on dira alors qu'il joue «en collaboration » avec l'architecte du labyrinthe; jouer à se faire peur) ou de tenter de les devancer («en compétition »; déjouer).

18. André Gardies, «Le pouvoir ludique de la focalisation », p. 139.

19. Par exemple chez Noël Carroll, une scène propose une question, puis le spectateur met en place un nombre d'hypothèses, puis une scène ultérieure apporte une réponse (confirme ou infirme une hypothèse) et, peut-être, pose une nouvelle question. Voir Noël Carroll, Mystifying Movies. Fads \& Fallacies in Contemporary Film Theory, New York, Columbia University Press, 1988.

20. Roger Caillois, Les jeux et les hommes. Le masque et le vertige, Paris, Éditions Gallimard, 1958, p. 41.

21. C'est probablement aussi le cas du spectateur qui n'en est pas à son premier visionnement, ou à celui qui refuse de se laisser prendre au jeu de la diégèse. Comme le théoricien, ils seraient alors en position d'observer le jeu et les différentes stratégies textuelles le soutenant. Voir la position du game-viewer chez Suits: "even the nonplaying reader can treat the story as a game: a game he is viewing rather than one he is playing". (Bernard Suits, «The Detective Story: A Case Study of Games in Literature », Revue canadienne de littérature comparée, vol. 12, n², juin 1985, p. 210) 
Or, nous l'avons mentionné, le volume (ou le monde diégétique qu'il propose), bien qu'en lui-même fermé et isolé, ne constitue pas la totalité de l'espace couvert par le système signifiant. Que se passe-t-il lorsque les indices et les pistes conduisent l'investigation du joueur hors du noyau, lorsque l'instance autoriale l'invite à quitter le centre? Si, par exemple, la phrase «C'était comme si personne n'avait entendu. » ne se présentait plus seule, mais accompagnée d'une note précisant son origine ${ }^{22}$, citation de la première phrase d'un roman? Il faudrait alors considérer ce système second, le roman en question, comme faisant partie du système signifiant complet, de la construction totale (du terrain de jeu) - et les questions ouvertes trouveraient peut-être alors réponses dans cet aiguillage qui, lui, n’aurait rien de linéaire. D'une façon ou d'une autre, cette expansion soudaine de l'espace à explorer implique à la fois que le signal émis déborde le volume en lui-même et que cette investigation (qui demeure un fait ludique) ne peut être conduite sous le joug de l'absorption diégétique. Ici, pour faire enquête, pour jouer, le lecteur ne peut que percevoir l'objet comme construit.

\section{UNE PÉRIPHÉRIE}

Tout texte, on le sait, se trouve ancré dans un réseau intertextuel composé d'un nombre multiple et instable de textes autres et dont il est le centre. Suivant Kristeva, "Il se crée, ainsi, autour du signifié poétique, un espace textuel multiple dont les éléments sont susceptibles d'être appliqués dans le texte poétique concret. Nous appellerons cet espace intertextuel ${ }^{23}{ }$. Le texte est centre, l'intertexte est périphérie; la signifiance est centripète.

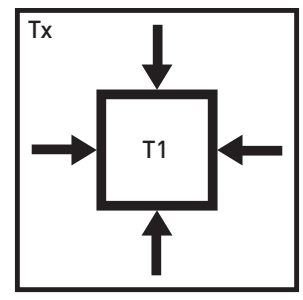

Fig. 2: Intertextualité (schéma - Intertexte [Tx]; Texte poétique concret [T1])

Le «texte poétique concret» correspond à ce que nous avons appelé le «noyau », le «volume» - le texte tel qu'en lui-même, centre du réseau intertextuel. La vaste majorité des croisements intertextuels est irrepérable; l'influence

22. Alain Robbe-Grillet, Le voyeur, Paris, Éditions de Minuit, 1955, p. 9.

23. Julia Kristeva, Sèméiotikè, p. 194 (nous soulignons). 
que ces croisements ont nécessairement sur la compréhension du texte par l'interprète est indéniable, mais ils ne font pas pour autant partie du système signifiant. Par contre, certains textes [ $\left.\mathrm{T}_{1}\right]$ présentent, dans cette myriade de carrefours, quelques croisements objectifs repérables. Il s'agit de relations textuelles concrètes, établissant effectivement une liaison entre deux systèmes textuels identifiés. Ces relations appartiennent au système, à la construction labyrinthique mise en place et proposée au lecteur-spectateur par l'instance autoriale. Elles font partie du système, comme ses mots, comme ses images, elles sont observables et disséquables - leur fonction est sujette à interprétation, mais les liaisons qu'elles mettent en place entre le «texte poétique concret» et d'autres entités textuelles identifiables forment le système signifiant global.

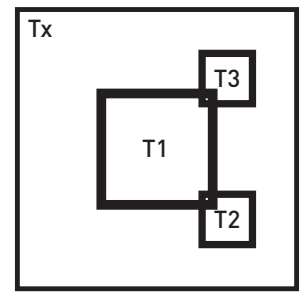

Fig. 3: Relations textuelles concrètes (schéma - Entités textuelles autres [T2], [ $\left.\mathrm{T}_{3}\right]$ )

"C'était comme si personne n'avait entendu. (Robbe-Grillet, Le voyeur, p. 9)» [T1], si on l'accepte comme «texte poétique» autonome, ne constitue qu'une partie (le centre ou la centrale, là où se rejoignent tous les canaux) de l'espace couvert par son système signifiant, puisque celui-ci renvoie à une entité textuelle autre, le roman cité [T2], qui se greffe à l'espace ouvert à l'investigation. À partir du centre, le lecteur-spectateur est convié à explorer les connexions mises en place par l'architecte du système, réseau intertextuel concret constitué par l'instance autoriale (terrain de jeu élargi). Examinons un exemple (réel) plus complexe (et moins bricolé).

\section{UN EXEMPLE: LA CENTRALE ET SES CANAUX HITCHCOCKIENS}

Après avoir été témoin de la mort d'une femme, un homme reconnaît le double (la doublure) de la victime et comprend que le drame ne s'est pas déroulé comme il l'avait d'abord perçu. Jake Scully fait enquête et, avec lui, le spectateur de Body Double (Brian De Palma, 1984). Le jeu est en place, l'instance autoriale trace une carte, établit un système et tente de réguler le savoir du spectateur, afin de le garder à sa place, dans la bonne voie; le joueur, quant à lui, s'il est « compétitif », devra voir à déjouer cette régulation, à spéculer et à devancer l'intrigue. Certains indices - l'idée du double, du rôle, du maquillage (Scully joue un vampire dans 
un film d'horreur) - lui permettront, peut-être, de mettre en doute l'identité du meurtrier et de formuler de nouvelles hypothèses. Le spectateur est absorbé dans la diégèse, à jouer au détective. Or, certaines des pistes qui lui sont proposées, s'il les perçoit et s'il choisit de les suivre, le conduiront hors de l'enclave diégétique - procédés réflexifs lui rappelant qu'il n'est pas un détective et donc, à un certain niveau, «briseurs de jeu». Par contre, bien qu'ils brisent le charme (qui n'est jamais que partiel) et qu'ils rappellent au spectateur sa position extra-diégétique, ces procédés réflexifs le cloisonnent instantanément dans un nouvel espace à investiguer: non plus les lieux de la diégèse de Body Double, mais l'ensemble Body Double/Vertigo, (Alfred Hitchcock, 1958), puis Body Double/Vertigo/Rear Window, (Alfred Hitchcock, 1954) où, suivant la figure 3, Body Double est le

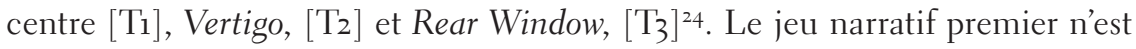
donc pas (vraiment) mis en échec par les bris narratifs, puisque c'est dans ce nouvel espace non linéaire que l'enquête mène le détective.

Lors de sa traversée matérielle des images et des sons du film de De Palma, le spectateur risque fort d'achopper sur un de ses plans, détonant par sa durée et sa mise en scène. Sur la plage, après avoir récupéré sa bourse des mains d'un voleur, Jake embrasse passionnément et longuement Gloria ${ }^{25}$. Contrairement à la phrase de Robbe-Grillet utilisée plus tôt, la référence à l'entité textuelle autre n'est pas ici initiée par une citation (de la reprise de la phraséologie exacte d'un texte source ${ }^{26}$ ), mais par une allusion au Vertigo de Hitchcock ${ }^{27}$. Le plan en

24. Les allusions à Hitchcock que l'on retrouve chez De Palma demeurent à notre avis parmi les plus explicitement ludiques et pertinentes à nos présentes considérations. Largement documentées, elles ne sont que rarement correctement identifiées - la majorité des commentateurs et critiques se contentant d'étiqueter les croisements de plagiats ou d'utilisations frauduleuses (voir par exemple Denyse Therrien, «Reconnaître des citations cinématographiques», dans Marie-Dominique Popelard et Anthony Wall (dirs.), Citer l'autre, Paris, Presses Sorbonne nouvelle, 2005, p. 145-146).

25. Il s'agit d'un des rares plans du film de plus d'une minute (1 m 15) et le seul de ceux-ci qui n’apportent aucun élément narratif nouveau (le couple s'embrasse déjà dans le plan précédent, et s'embrasse encore dans le plan suivant). Cette agrammaticalité jure avec l'ensemble: "These "ungrammaticalities" prompt him [le lecteur spectateur] to look for a solution elsewhere, that is, in the intertext. (Michael Riffaterre, «The Interpretant in Literary Semiotics ", American Journal of Semiotics, vol. 3, n 4, 1985, p. 42)

26. Voir Claudette Sartiliot, Citation and Modernity. Derrida, Joyce and Brecht, Norman, University of Oklahoma Press, 1993, p. 22 : «Indeed, citation, as opposed to allusion, for example, denotes of the exact phraseology of the other. "

27. On dira donc que La jetée (Chris Marker, 1962) fait également allusion à Vertigo, mais que Twelve Monkeys (Terry Gilliam, 1995) le cite, puisqu'il en réactualise les images, le son et la musique (citation qui est, néanmoins, allusive). 
question, marqueur de l'allusion, est réalisé en miroir d’un plan du film-référent: le mouvement de caméra circulaire autour du couple qui s'embrasse, comme la musique de Pino Donaggio, sont calqués sur le travail de Hitchcock et Bernard Hermann. Bien sûr, le marqueur peut être aplati dans la diégèse du film et le spectateur peut lire la scène sans percevoir le canal ouvert par l'instance autoriale. C'est dire que le marqueur est signifiant et acceptable dans le défilement narratif de Body Double, mais qu'il pointe également à une signifiance extérieure à ce défilement ${ }^{28}$. Empruntant aux schémas de l'allusion littéraire proposés par Ziva Ben-Porat ${ }^{29}$ et Anthony Johnson ${ }^{30}$, nous proposons cette combinaison complémentaire aux schémas précédemment utilisés, à notre avis plus compréhensible et plus compréhensive du procédé :

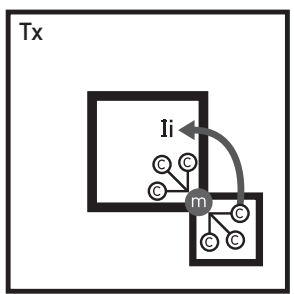

Fig. 4. Allusion (schéma-Marqueur [m]; Caractéristiques [c]; Interprétation intertextuelle [Ii])

Le marqueur $[\mathrm{m}]$ se superpose à l'élément marqué du système auquel l'allusion pointe. S'ajoutent aux caractéristiques [c] lui étant propres dans le système hôte, de nouvelles caractéristiques potentielles, celles que possède l'élément marqué dans le système autre (et, par extension, celles que possède ce système lui-même). Le lecteur-spectateur percevant l'allusion est invité à tenir compte de ces nouvelles caractéristiques potentielles et, possiblement, à en actualiser un certain nombre dans le système hôte - opération lui permettant une interprétation intertextuelle [Ii] du marqueur (une interprétation aplatissant le marqueur dans le système hôte et qui ne tiendrait compte que de ses caractéristiques propres est dite locale). Considérant ce nouvel espace augmenté, et considérant les différentes caractéristiques s'appliquant au couple du film de Hitchcock,

28. Voir Carmela Perri, «On Alluding», Poetics, vol. 7, n 3, septembre 1978, p. 295 : "an allusion refers at least doubly: the sign of the allusion-marker refers within its text's world as well as allusively, to some referent outside this text ».

29. Ziva Ben-Porat, «The poetics of literary allusion», PTL: A Journal for Descriptive Poetics and Theory of Literature, vol. 1, $\mathrm{n}^{\circ} 1$, janvier 1976, p. 105-107; p. 115.

30. Anthony Johnson, "Allusion in Poetry», PTL: A Journal for Descriptive Poetics and Theory of Literature, vol. $1, \mathrm{n}^{\circ}$ 3, octobre 1976, p. 579-587. 
le spectateur a beau jeu de chercher des indices, dans Vertigo, s'appliquant à l'intrigue de Body Double. Sur cette voie, il pourrait, peut-être, jaugeant le fait que Scully est grosso modo un double de Scottie (ils sont tous deux phobiques et incapables de pratiquer leur métier; tous deux sont sous le charme d'une femme mariée qu'ils ne connaissent guère; tous deux sont témoins de sa mort), postuler que Scully pourrait avoir, lui aussi, été le pion et l'alibi du meurtrier : le mari.

De la même façon, des allusions à Rear Window ouvrent le système signifiant dans une autre direction, sur un autre espace à explorer, et à nouveau le spectateur jouant au détective peut y dénicher des réponses avant qu'elles n'apparaissent dans le défilement narratif de Body Double: Jeff, comme Scully, utilise un appareil optique pour épier ses voisins, persuadé qu'une femme a été assassinée, il fait enquête pour prouver l'identité du meurtrier (le mari). Bien sûr il ne s'agit là que d'une façon de jouer le film et d'utiliser la carte tracée par le système signifiant, la plus banale sans doute car la plus fidèle aux directives de l'instance autoriale. Le film de De Palma soulève d'autres questions que celles tourmentant son personnage principal (des questions sur la facticité, le double, le regard et le voyeurisme, etc.) et comme aucune absorption diégétique n'est nécessaire pour jouer dans ce nouvel espace, le spectateur est également invité à parcourir celui-ci au-delà de ces récits - les croisements n'ouvrent pas uniquement sur les films de Hitchcock eux-mêmes, sur leurs intrigues, mais sur toute la littérature qui les entoure. Il y a là, pour chacun, amplement de quoi jouer.

L'insertion par le personnage de Fight Club (David Fincher, 1999) d'un gros plan de sexe masculin dans un film pour enfants, ainsi que l'apparition de ce gros plan dans le montage du film de Fincher, fonctionnent de la même façon: elles sont des allusions à un insert de même nature utilisé par Ingmar Bergman dans Persona (1966), elles ouvrent l'espace à investiguer et invitent le spectateur à considérer le film de Bergman dans sa lecture de celui de Fincher. Parmi les nombreux éléments plastiques et narratifs ainsi offerts à lui, le spectateur a beau jeu de noter que l'une des caractéristiques fortes de Persona, «c'est d'avoir seulement deux personnages, et que chacun soit le démon de l'autre; deux personnages aussi proches l'un de l'autre que des doubles virtuels ${ }^{31}{ }^{1}$ et par la suite d'appliquer cette caractéristique à sa lecture de Fight Club, perçant ainsi l'ambiguïté identitaire de ses deux personnages masculins. Toujours dans l'optique du double, une allusion à Lost Highway (David Lynch, 1997) dans le film Caché de Michael Haneke (2005) amènera peut-être le spectateur à chercher le coupable non pas dans l'entourage du personnage principal, mais dans sa propre psyché.

31. Jacques Aumont, Ingmar Bergman: «Mes films sont l'explication de mes images », Paris, Cahiers du cinéma, 2003, p. 177. 
Les différents types de relations textuelles concrètes (les renvois allusifs et parodiques, le plagiat, même la citation canonique et sa fanfare de marqueurs) peuvent être aplatis par une lecture qui ne considérerait que le centre du système signifiant. Il appartient au lecteur-spectateur de re-tracer le petit réseau textuel concret que lui propose l'instance autoriale. Comme cette tâche repose sur une compétence de lecture, une attention et des connaissances préalables variables, il est peu probable (voire impensable) - car le lecteur-spectateur trace sa propre carte des lieux - que le système signifiant, édifice de l'instance autoriale, soit exploré adéquatement et exhaustivement. Il faudrait d'abord que le signal reçu corresponde au signal émis.

\section{UNE ZONE HORS JEU}

Il y a, nous l'avons vu, autour du système signifiant (et donc, au-delà des relations textuelles concrètes), un espace textuel multiple, l'intertexte, générant une quantité indéterminable de croisements influençant eux aussi la construction et l'interprétation du système. Relations textuelles non concrètes, ou diffuses, elles ne mettent pas en relation effective le système signifiant avec d'autres entités textuelles: le pastiche ne lie pas le système à un texte identifié (et identifiable), mais à un groupe de textes plus ou moins vaste; le lieu commun est un raccourci narratif qui prend sens du fait qu'il existe dans différents systèmes; le cliché est un identifiant générique. Par exemple, le recours dans un film narratif à un lieu commun (le commissariat de police bondé d'une mégapole américaine; l'autobus jaune venant prendre les enfants le matin) ne pose pas une référence concrète à une autre oeuvre identifiée employant elle aussi la même figure, mais utilise plutôt l'épaisseur signifiante de celle-ci, accumulée au fil de ses apparitions dans différents systèmes. Ces croisements diffus et irrepérables, rappelant d'autres systèmes signifiants, rappelant des expériences ou des connaissances emmagasinées, suscitent différentes inférences du lecteur-spectateur permettant et favorisant l'étendue (illimitée) de ses interprétations, «au-delà des limites de l'œuvre comme telle ${ }^{2}$ ».

Non concrets, non objectifs, les multiples croisements potentiels occupant le réseau intertextuel sont par contre objectivables. La reconstruction du système signifiant par le lecteur/spectateur est sujette à être parasitée par certaines connections intertextuelles n'appartenant pas au signal émis. Ainsi, ce n'est pas l'espace aménagé par l'instance autoriale que le lecteur-spectateur explore (et

32. Richard Shusterman, Sous l'interprétation, trad. Jean-Pierre Cometti, Combas, Paris, Éditions de l'éclat, 1994, p. 13. 
interprète), mais sa propre reformulation du système. Comme nous l'avons vu, cette reformulation peut bien sûr être estropiée - s'il a aplati les marqueurs d'allusions dans sa lecture narrative de Body Double, les espaces sur lesquels ceux-ci ouvraient n'existent tout simplement pas dans cette reformulation - mais elle peut également être élargie, et c'est cet élargissement intertextuel du système signifiant lors de sa réception, par croisements objectivés, qui nous intéressera maintenant.

Toute relation textuelle non concrète est un carrefour intertextuel multiple, un échangeur où ça circule, où il n'y a pas de liaison arrêtée (où il n'y a pas de liaison arrêtée, pour les avoir toutes). Un tel carrefour perce une brèche dans le système signifiant, non pas une ouverture donnant sur un autre système comme le ferait une relation textuelle concrète, mais un embranchement à plusieurs voies, où plusieurs possibilités de connexion se présentent, sans s'attacher à aucune destination fixe. Il y a, au sens mécanique du terme, du jeu dans le système. Cette infinité de possibilités crée, à la réception, un bourdonnement encyclopédique autour du système signifiant, du bruit, et - par confusion, par errance ou par pur plaisir ludique - le lecteur-spectateur est susceptible de combler la première venue de ces brèches par un canal, en y arrêtant la circulation sur une référence fixe ayant pour lui une résonance particulièrement accentuée. Se faisant, il joue [avec] le texte 33 , il en modifie l'aire de jeu et l'ouvre à de nouvelles interprétations - celles-ci, bien qu'elles puissent être constructives et générer une nouvelle compréhension du système, ne s'y appliquent pas directement, mais impliquent qu'il y ait valeur ajoutée. Nous dirons donc qu'il s'agit en fait de (sur)interprétations du système.

Michel Serres utilise les métaphores du joker et du domino blanc (deux renvois au jeu dans le système) afin de qualifier ces points de flottement où les possibilités de connexion au système se présentent. «Le joker, au lieu de la bifurcation, la rend possible par le confluent des valeurs qu'il assure. Il est, à la fois, ce qui est déjà dit et ce qui va se dire. Il est à deux, à trois, ou à plusieurs valeurs, selon la complexité de la connexion34. »C'est l'interchangeabilité qui caractérise la figure du joker: sa valeur est changeante, il peut assumer celle des différents éléments

33. Le lecteur/spectateur se libère ainsi de la directivité imposée par la stratégie textuelle qui, comme l'animateur imposant des règles à l'enfant, le brime dans sa capacité de jouer de façon créative (voir Donald Winnicott, Playing and Reality, p. 50). Cette possibilité de participer de façon créative à la composition du système signifiant amène un certain équilibre entre les joueurs, parité nécessaire au jeu (voir Elisabeth S. Bruss, «The Game of Literature and Some Literary Games », New Literary History, vol. 9, n 1, automne 1977, p. 154: «a game, by definition, is the encounter between equally matched and equally creative participants »).

34. Michel Serres, Le parasite, Paris, Hachette Littératures, coll. «Pluriel», 1997 [1980], p. 290. 
d'un ensemble donné. Dans un jeu de cartes, «[1]e joker est roi ou valet, il est l'as ou le sept, ou le deux, à loisir35». Sa valeur dépend des choix du joueur.

À sa reformulation, tous les éléments d'un système signifiant, toutes les images et les sons d'un film, tous les mots d'un texte sont susceptibles de faire brèche, d'être jokers. On l'a vu, chacun de ces éléments donne sur un paradigme - d'une part linguistique (ou iconique), d'autre part encyclopédique et s'avère potentiellement polysémique. C'est le rôle de la stratégie textuelle de mettre en place une directivité (souvent par l'usage de conventions textuelles) ou d'en assurer l'absence, faisant en sorte de garder le lecteur-spectateur en-jeu, ou au contraire de l'égarer volontairement ${ }^{36}$. L'ambiguïté d'un système est en grande partie relative au nombre de jokers (de carrefours sémiotiques, d'éléments interchangeables) ${ }^{37}$ qu'il comporte, mais l'instance autoriale perd tout contrôle sur le signal émis une fois celui-ci en jeu. L'autre joueur, de créativité égale, redistribue les cartes.

\section{DU BRUIT ET DES INTERFÉRENCES}

«Le bruit est un joker. Il a au moins deux valeurs, comme le tiers: une valeur de destruction, une valeur de construction $3^{8}$. » Chaque élément est susceptible de générer un croisement non concret, d'être utilisé comme joker, du fait que le système n'est pas maître du bruit accompagnant sa réception. Il y a de la friture, du bruit, sur la ligne - c'est toute la résonance encyclopédique et intertextuelle des éléments du système qui risque de faire interférence sur celui-ci.

La notion d'interférence a l'avantage de comprendre d'un coup le jeu des interrelations qui ouvrent les régions les unes aux autres, l'unité de circulation [...] qui résulte de ce jeu, le transport en général et la difficulté de lui assigner une source autochtone. Elle restitue, enfin, l'image du réseau, et laisse une ouverture indéfinie au champ global du savoir par intersections continuées. Mais elle ruine, à tout jamais, l'idée de référence39.

35. Michel Serres, Le parasite, p. 286.

36. Évidemment on pensera dans le premier cas au texte juridique, et dans le second, au poème dadaïste.

37. «Soit donc l'univers du discours. On peut ordonner ledit univers selon la distribution des jokers. S'il y en a peu dans une coupe ou dans une séquence, la détermination est forte et la contrainte règne, on est assez voisin de la monosémie. [...] Allez au maximum, allez à saturation. La polysémie envahit l'espace, la multivalence, l'équivocité. Au voisinage de la fin, c'est le monde du rêve. » (Michel Serres, Le parasite, p. 292)

38. Michel Serres, Le parasite, p. 123.

39. Michel Serres, L'interférence, Paris, Éditions de Minuit, 1972, p. 63. 
Ces croisements interférentiels, contrairement aux relations textuelles concrètes, fonctionnent sans référence. Le système hôte lui-même ne contient aucun renvoi, il ne pointe pas vers un système autre. C'est une relation sans origine ni fin, c'est la pluralité opérante ${ }^{\circ}$ du système. Une interférence se produit lorsque, dans la densité intertextuelle, un croisement se trouve concrétisé à la réception, sans que la stratégie textuelle ne le commande. La brèche dans le système signifiant se trouve ainsi comblée alors qu'elle ne demandait pas à l'être, alors qu'elle aspirait à une circulation sémiotique fluide. Les sources intertextuelles non concrètes de la signifiance du système ne peuvent être retracées et inventoriées, elles sont nécessairement multiples et instables. Une source identifiée (comme telle) par le lecteur-spectateur, si elle n'est pas associée à une relation textuelle concrète, risque fort de faire interférence sur sa réception : liée au système signifiant, elle en augmente l'espace, mais la reformulation est une création du lecteur-spectateur, un nouvel espace pour jouer, une nouvelle configuration à (sur)interpréter. Dans le réseau interférentiel, le croisement objectif est absent. Les textes se cognent et se superposent ${ }^{41}$, ils donnent l'impression de se croiser. Ces croisements subjectifs se concrétisent effectivement, mais à la réception uniquement: ils n’appartiennent pas au signal émis. Il n'y a dans le système que le jeu, que le joker, le point de flottement ayant généré la liaison - la figure est là. Le croisement n'est pas objectif, mais il est objectivable.

\section{UN EXEMPLE : POSSESSION(S)}

Après s'être aventurée seule dans un tunnel, Anna est frappée d'une crise soudaine, se jetant sans raison apparente contre le mur et le sol. La scène de Possession (Andrzej Zulawski, 1981) est en soi ambiguë et ouverte à différentes interprétations : simple crise psychotique, genèse de la possession du personnage, fausse couche ou enfantement de la créature tentaculaire, etc. Ces interprétations sont générées par les images et les sons appartenant au système signifiant, leurs différentes combinaisons, ainsi que toutes les associations non concrètes effectuées par le spectateur dans les champs encyclopédiques et intertextuels. Par exemple, les cris saccadés d'Anna, les positions qu'elle adopte, les liquides laiteux qui lui couvrent le corps peuvent certes résonner, dans ces champs, comme étant de nature sexuelle. L'interprète ne quitte pas alors le terrain de jeu, mis en place par l'instance autoriale, et les différentes interprétations auxquelles il parvient, bien qu'elles soient partiellement le produit d'expériences ou de connaissances

40. Voir Julia Kristeva, Sèméiotikè, p. 284.

41. «Interférer, c'est frapper, se frapper contre. » (Daniel Vaillancourt, «L'interférence relue et visitée », Protée, «Les interférences », vol. 24, n² 2, automne 1996, p. 9) 
emmagasinées, demeurent néanmoins suggérées par le système signifiant ${ }^{2}$. Elles sont engendrées par les éléments le constituant.

Le spectateur qui, lui, dans cette résonance diffuse (dans ce bruit de fond), identifie des sources ponctuelles et précises et trace des canaux de transport signifiant, aménage pour sa part un petit réseau ne correspondant pas au système signifiant tel qu'émis. En ce sens, il est hors-jeu (et non pas hors-du-jeu), uniquement parce qu'il joue selon des règles différentes de celles proposées par la stratégie textuelle, parce qu'il s'aventure (ou s'égare) concrètement hors des limites établies du terrain de jeu. Son jeu n'est plus assujetti aux directives de l'animateur, il est créatif - il a au moins deux valeurs : une valeur de destruction, une valeur de construction, «Le bruit est la fin d'un système et la formation d'un nouveau 43 ». Et si le système premier est l'édifice de l'instance autoriale, sa reformulation en un nouveau système est l'édifice du joueur, du lecteur-spectateur. Les (sur)interprétations générées par ce nouveau système sont bien sûr susceptibles d'être en tous points applicables au système signifiant premier, elles peuvent en enrichir la lecture comme elles peuvent confirmer des interprétations qui auraient pu être nées de celui-ci sans valeur ajoutée. Il ne faut donc pas entendre ici (sur)interprétation comme divagation, mais bien comme interprétation d'un objet qui n'est pas le système signifiant en lui-même; "au sens où la surinterprétation serait l'interprétation adéquate à tout phénomène surdéterminét4. »

La jeune femme aux cheveux noirs, la robe, le sac à main, le tunnel, l'éclairage provenant du plafond à intervalle régulier, les chocs contre le mur et le sol, les cris et les positions du corps, les couleurs saturées de la scène (bleu dans un cas, rouge dans l'autre), voire l'utilisation d'une actrice de langue étrangère reconnue pour ses charmes: tous ces éléments sont susceptibles de trouver écho dans l'intertexte du système. Aucun renvoi référentiel ne lie Possession à Irréversible (Gaspar Noé, 2002), mais il y a une nette possibilité interférentielle entre les deux films, dans un sens comme dans l'autre (comme le point de croisement n'est pas concret, il n'est pas directif et ne dépend que de son édification). Architecte de ce croisement ajouté (de sa concrétisation), le spectateur peut interpréter le système signifiant qui en résulte: Anna est violée dans le tunnel et c'est là l'origine de son déséquilibre psychologique; Anna est violée, à un niveau psychologique ou symbolique, par la créature qui la possède; Alex, la jeune femme violée dans

42. «L'interprète, au moment même où il s'abandonne au libre jeu des réactions qui lui sont suggérées, revient à l'objet pour y trouver l'origine de la suggestion. » (Umberto Eco, L'œuvre ouverte, p. 139)

43. Michel Serres, Le parasite, p. 122.

44. Georges Didi-Huberman, Ouvrir Vénus. Nudité, rêve, cruauté, Paris, Éditions Gallimard, coll. «Le temps des images», 1999, p. 32. 
le tunnel d'Irréversible, est victime d'une possession maléfique (le croisement utilisé comme métaphore), etc. Ces (sur)interprétations sont applicables et (plus ou moins) pertinentes au système signifiant premier considéré individuellement, certaines d'entre elles auraient clairement pu naître de sa seule réception, mais générées ou justifiées par un croisement interférentiel, elles ne peuvent être considérées comme de simples interprétations. L'interférence elle-même est, au sein du système signifiant, asignifiante. Elle est le fruit du hasard, de la libre association, toute signifiance que l'on pourrait attribuer à un tel croisement origine plutôt de son architecte, de la créativité du joueur.

\section{DE L'ENTROPIE}

Le système interférant brouille la réception, il se frappe au canal de communication entre le système signifiant et l'interprète; l'interférence n'appartient pas au système signifiant premier, mais à la relation le liant au lecteur-spectateur. Le système interférant fait du bruit, il risque de perturber la lecture. Afin d'illustrer cette perturbation signifiante, nous proposons une variation du schéma de la communication de Shannon (une reformulation):

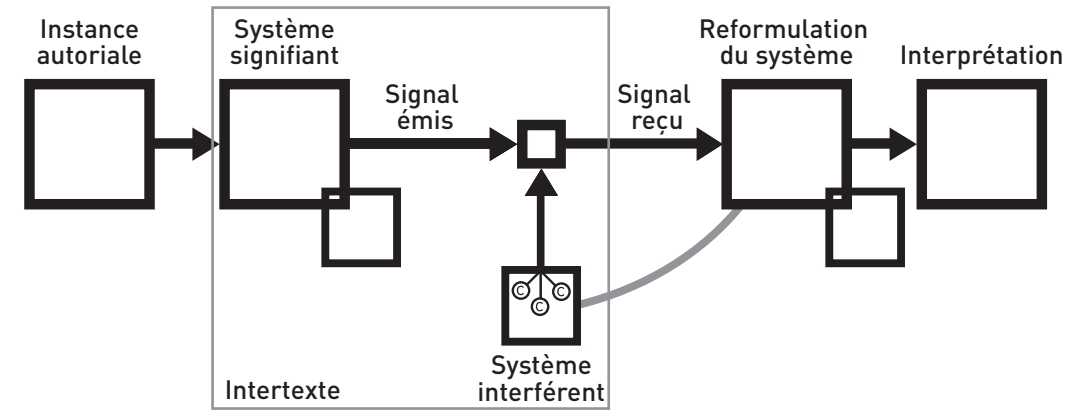

Fig. 5. Interférence (schéma - Interférence [i]; (Sur)interprétation [(S)I])

Comme nous l'avons noté plus haut, schématiser le réseau interférentiel est problématique : c'est l'arrêter, c'est nier son instabilité et sa mouvance constante c'est une simplification qui ne tient pas compte de la fluidité du réseau ni de la multiplicité des croisements possibles. Le graphe idéal devrait être tridimensionnel, élastique et en mouvement: les régions du réseau se heurtant les unes aux autres et se superposant (au gré des mouvements aléatoires du graphe et de la position de l'observateur). Dans ce schéma, le système interférant tient lieu de la source du bruit, notons cependant qu'il n'en est pas l'unique source et que toutes les sources de bruit ne sont pas susceptibles de faire interférence - certaines, indistinctes ou multiples, sont proprement irrepérables, d'autres se produisent dans l'échange à un niveau technique et ne nous concernent guère. Bien sûr, 
la reformulation du système, comme toute forme de traduction, est déjà une interprétation, elle en constitue véritablement la première phase ${ }^{45}$, celle sur laquelle s'appuient les suivantes. Il y a du jeu dans le système signifiant, dans la stratégie textuelle - un indésirable est susceptible de s'introduire dans l'engrenage, de le faire dérailler. L'interférence comme phénomène physique, superposition d'oscillations ou d'ondes de même nature et de fréquences égales ou voisines, est un parasite ${ }^{6}$, une source d'entropie:

La théorie de l'information, dans son étude de la transmission, considère précisément les messages comme des systèmes organisés, régis par des lois fixes de probabilité, mais dans lesquels peut s'introduire, sous forme de perturbation venant de l'extérieur ou d'atténuation du texte même (tous ces éléments entrant dans la catégorie du «bruit»), un pourcentage de désordre, donc d'usure de la communication et d'augmentation de l'entropie 47.

Il est donc question de désordre, de la destruction d'un système, mais de la constitution d'un ordre nouveau, différent et décentré, affranchi de la rigidité des échanges référentiels unidirectionnels. Le réseau interférentiel ouvre le système signifiant à l'infinité de ses carrefours. Il amène à considérer les croisements qui y sont latents, qui n'ont pas été actualisés par l'instance autoriale, mais qui le seront potentiellement, par d'autres instances jouant avec le système. Il amène à une nouvelle compréhension de l'échange et de la circulation intertextuelle ${ }^{4}$.

\section{DU PLAISIR}

Le réseau interférentiel convoque des relations non hiérarchiques, il est non centré, ou décentré, ou a son centre partout 49 . En ce sens il se trouve plus près des conceptions de l'intertextualité de Barthes ou de Derrida que de celles de

45. Voir Hans Robert Jauss, Pour une herméneutique littéraire, trad. Maurice Jacob, Paris, Éditions Gallimard, coll. «Bibliothèque des idées», 1982 [1977], p. 383: «La première étape [de l'interprétation] devait suivre pas à pas la perception esthétique du lecteur jusqu'à ce que, avec le dernier vers, la structure du poème, à défaut peut-être du sens, lui apparaisse comme formant un tout. »

46. Voir Michel Serres, Le parasite, p. 256: «Parasite. Le préfixe para signifie voisin, à côté de, mesure une distance, comme un écart léger. »

47. Umberto Eco, L’uvre ouverte, p. 77.

48. «À prendre acte, tout d'abord, du pluralisme, du relativisme des références possibles, du caractère décisoire ou arbitraire de leur désignation. À élaborer, ensuite et surtout, un type de pensée dé-centrée, non référenciée, où l'essentiel n’est pas de se frayer un chemin vers un ancrage supposé, et toujours présupposé, quel qu'il soit, mais de se poser la question du chemin lui-même, du transfert et du transport. » (Michel Serres, L'interférence, p. 144-145)

49. Michel Serres, L'interférence, p. 63. 
Genette ou de Riffaterre. Un croisement objectivé par le lecteur-spectateur lors de la reformulation d'un système signifiant recentre le réseau autour de ce système, lui donnant préséance dans la signifiance globale de l'embranchement. Ce faisant, il balise un nouvel espace qui, à partir de ce noyau, invite au jeu. La perception d'une interférence, et c'est ce qui nous intéressait ici, est proprement une incitation à la (sur)interprétation. Il n'est cependant pas nécessaire d'exploiter la liaison établie, de la (sur)interpréter, pour en tirer un plaisir ludique. Ce plaisir se fonde en grande partie dans l'établissement du croisement lui-même et le lecteur-spectateur peut très bien s'en tenir à la concrétisation de croisements absents. Son jeu serait alors plus épistémologique ou cinéphilique que narratif ou prospecteur. Il y a par exemple certainement du plaisir à prendre d'un croisement perçu entre The Ladies Man (Jerry Lewis, 1961) et Spider-Man (Sam Raimi, $2002)^{50}$, sans pour autant chercher à en tirer une explication narrative dans l'un ou l'autre des systèmes signifiants interférants.
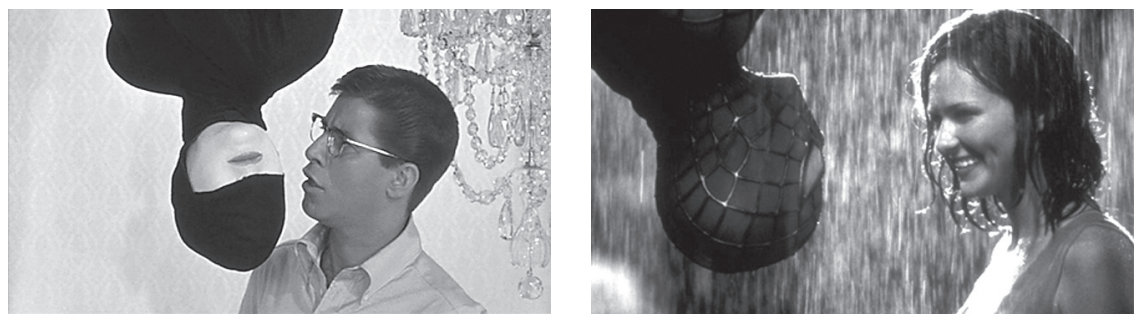

Fig. 6. Jerry Lewis, The Ladies Man, 1961 (C) Paramount Pictures; Sam Raimi, Spider-Man, 2002 (c) Sony Pictures.

On ne saurait le dire plus simplement: «L'interférence est, proprement, la réduction de la différence ${ }^{51} »$. Et c'est cette réduction qui est génératrice de jeu, au sens mécanique (puisqu'elle fait brèche dans le système signifiant, instituant un espace de flottement à combler), comme au sens ludique car, comme pour la parodie et l'allusion (où la réduction participe au contrat de lecture), c'est d'abord dans la reconnaissance (de la ressemblance ou de la différence réduite) que se fonde le plaisir ludique de l'interférence.

50. Nous tenons à remercier Jean-Marc Limoges, un spectateur extraordinaire, pour cet exemple d'interférence.

51. Michel Serres, L'interférence, p. 40. 